\title{
ЦЕННОСТИ, СОЦИАЛЬНАЯ ДИСТАНЦИЯ И УСТАНОВКИ ПО ОТНОШЕНИЮ К МИГРАЦИИ: КРОСС-КУЛЬТУРНОЕ ИССЛЕДОВАНИЕ В БЕЛЬГИИ, ГЕРМАНИИ, ФРАНЦИИ И НИДЕРЛАНДАХ
}

\begin{abstract}
Д.С. ГРИГОРЬЕВ
${ }^{a}$ Национальный исследовательский университет «Высшая школа экономики», 101000, Россия, Москва, ул. Мясницкая, д. 20

\section{Резюме}

В статье рассмотрена взаимосвязь индивидуальных ценностей, социальной дистанции и позитивных и негативных установок по отношению к миграции, целью настоящего исследования была проверка культурно-универсального характера этих связей. Ранее исследователями крайне редко рассматривались оба аспекта отношения к мигрантам: восприятие мигрантов принимающим обществом и мнение принимающего общества относительно миграционной политики. В данном исследовании были рассмотрены оба этих аспекта в форме социальной дистанции и трех установок по отношению к миграции. С помощью анализа данных 7-го раунда Европейского социального исследования (European Social Survey, ESS) за 2014 г. для четырех европейских стран (Бельгии, Германии, Франции и Нидерландов) после проверки метрической (сильной факторной) инвариантности шкал и их надежности с использованием моделирования структурными уравнениями было выявлено, что, как и предполагалось в гипотезах исследования, социальная дистанция положительно связана с ценностями сохранения и отрицательно с ценностями открытости к изменениям, а также положительно связана с негативными установками по отношению к миграции (восприятие миграции как угрозы и необходимость некого миграционного ценза) и отрицательно с положительными установками (желательность миграции), при этом данный характер связей носит культурно-универсальный характер. Также, помимо этих универсальных связей, можно отметить, что многие другие связи в модели носят культурно-специфический характер, особенно примечательным является эффект полной медиации социальной дистанцией некоторых связей между отдельными ценностями и отдельными установками на разных выборках. Полученные результаты полезны для понимания некоторых аспектов межкультурных отношений.
\end{abstract}

Ключевые слова: социальная дистанция, ценности, межэтнические отношения, иммиграция, кросс-культурная психология.

Статья подготовлена в ходе проведения исследования в рамках Программы фундаментальных исследований Национального исследовательского университета «Высшая школа экономики» (НИУ ВШЭ) и с использованием средств субсидии в рамках государственной поддержки ведущих университетов Российской Федерации «5-100». 


\section{Введение}

На сегодняшний день, несмотря на многолетние попытки проведения политики мультикультурализма в некоторых странах, можно констатировать, что негативное отношение к культурным, религиозным и этническим меньшинствам, трудовым мигрантам и политическим беженцам весьма распространено в демократических обществах (Halperin et al., 2007). В связи с последними событиями в Европе, такими как массовые нападения мигрантов на женщин в немецком Кельне и Швеции, при непрекращающемся потоке беженцев данная проблема только обострится, поскольку дискриминация, негативные установки и эмоции по отношению к мигрантам со стороны принимающего населения связаны с восприятием мигрантов в качестве угрозы (Stephan, Stephan, 2001).

Обычно в исследованиях различают два аспекта отношения к мигрантам: восприятие мигрантов принимающим обществом и мнение принимающего общества относительно миграционной политики (Tartakovs$\mathrm{ky}$, Walsh, 2016). В настоящем исследовании будут рассмотрены оба этих аспекта в форме социальной дистанции и установок по отношению к миграции.

Опросы общественного мнения во всем мире неоднократно показывали сильное восприятие негативных аспектов миграции, таких как социально-экономическое ухудшение и увеличение преступности (Fitzgerald et al., 2012; Higgins et al., 2010; Simon, Sikich, 2007), но есть также и исследования, показывающие положительное отношение к мигрантам: существуют общества, значительная часть которых поддерживает постоянную миграцию в страну (Lee, Fiske, 2006; Leong, 2008). Таким образом, в комплексную теорию, объясняющую отношение принимающего общества к миграции, следует наряду с восприятием миграции как угрозы также включать и позитивные аспекты ее восприятия (Tartakovsky, Walsh, 2016). В данной статье будет рассматриваться сильно негативное отношение к миграции (миграция как угроза), слабо негативное отношение к миграции, т.е. селективный подход к мигрантам, принятие только тех мигрантов, которые соответствуют определенным условиям (миграционный ценз), и положительное отношение к миграции (желательность миграции).

\section{Социальная дистанция как проявление предрассудков по отношению к мигрантам}

По мнению многих исследователей межэтнических отношений, социальная дистанция является общепринятой обобщенной мерой этнических предрассудков (Weaver, 2008 ) и одним из основных инструментов в исследованиях межгрупповых отношений, особенно в социально-психологических исследованиях этнических групп или рас (Laumann, 1965), при этом она является еще и наиболее изученным проявлением предрассудков (Duckitt, 2003).

Понятие социальной дистанции, впервые рассмотренное американскими социологами Р. Парком и Э. Богардусом, предполагает существование некого социального «расстояния» 
между людьми, которое можно измерить, как, например, физическое расстояние (Kim et al., 2015), оно определяется как степень, в которой люди хотели бы избежать тесного контакта между собой и членами различных социальных, расовых, этнических или национальных групп (Park, 1924; Bogardus, 1925). Сoциальная дистанция отражает некое чувство готовности общения с членом аутгруппы (Williams, 1964), она также свидетельствует, насколько приемлемо или, наоборот, нежелательно присутствие различных этнических групп в обществе (Marger, 1994). Часто социальная дистанция мотивирована чувством дискомфорта при контакте с членами аутгруппы, при этом само по себе декларирование этнического неравенства явно не утверждается (Kleinpenning, Наgendoorn, 1993). Социальная дистанция является своего рода границей, разделяющей ин- и аутгруппу (Kim et al., 2015).

Социальная дистанция, зависящая от социальной структуры, может быть измерена как объективный показатель (Simmel, 1950; Kadushin, 1962). Чтобы избежать путаницы, для ее психологического раскрытия некоторые исследователи используют термин «субъективная социальная дистанция» (Laumann, 1965).

Конкуренция на политических, социальных, экономических или культурных основаниях отмечается в большей части современных исследований как одна из основных причин негативных установок и социальной дистанции (напр., см.: Sniderman et al., 2004), что, в свою очередь, связано с уровнем воспринимаемой угрозы, которая, по мне- нию многих исследователей, представляется как самый надежный предиктор негативных установок в сфере межгрупповых отношений (Stephan, Stephan, 2001). Воспринимаемая угроза, прежде всего, выражается в когнитивной оценке аутгруппы как мешающей достижению ингрупповых или/и индивидуальных целей, что впоследствии может усилить социальную дистанцию или даже привести к насильственным действиям по отношению к представителям аутгруппы (Pettigrew, 2003).

При этом также целесообразным является поиск и индивидуальных черт личности, которыми можно объяснить негативное отношение к меньшинствам (Halperin et al., 2007). В предыдущих исследованиях социальной дистанции по отношению к группам меньшинств или мигрантов упоминаются гражданская идентичность, политическая ориентация и ценности как факторы, которые влияют на социальную дистанцию, при этом связь с социально-демографическими переменными (пол, возраст, образование и пр.) представляется разными авторами неоднозначно (Kim et al., 2015).

Таким образом, рассматривая социальную дистанцию, можно лучше понять, как граница между ин- и аутгруппой будет связана с отношением к различной политике по принятию мигрантов со стороны населения.

\section{Ценности и отношение к миграции}

Согласно теории ценностей Ш. Шварца (Schwartz et al., 2012; Schwartz, Butenko, 2014), ценности 
(или ценностные ориентации) представляют собой желательные трансситуационные цели, различающиеся по важности и служащие руководящими принципами в жизни людей. В своей последней редакции (Schwartz, Butenko, 2014) теория определяет полный набор из 19 мотивационно различных ценностей (безопасность, конформность, гедонизм, стимуляция, самостоятельность и т.д.). Ценности находятся в различных отношениях между собой и могут складываться более крупные ценностные блоки высшего порядка: Самопреодоление, Самоутверждение, Открытость изменениям и Сохранение.

Предполагается, что человеческие ценности, будучи некими универсальными абстрактными принципами, определяют конкретные установки и поведение, ценности привносят некоторую иерархию значимости для убеждений при условии, что общая оценка объекта уже выполнена, также они влияют на установки по отношению к таким объектам, которые связаны с достижением мотивационных целей, на которые направлены соответствующие ценности, т.е., например, в случае, когда миграция мешает или, наоборот, способствует достижению некоторых мотивационных целей, эта ситуация и будет определять связь между конкретными ценностями и установками по отношению к миграции (Davidov et al., 2008).

Исследователи предположили, что определенные установки по отношению к меньшинствам соответствуют одним и противоречат другим ценностям (Tartakovsky, Walsh, 2016), например, ценности сохранения (особенно безопасность) могут быть связаны с негативными установками к группам меньшинств, в то время как ценности открытости к изменениям (особенно самостоятельность и стимуляция) могут быть связаны с положительными установками по отношению к этим группам (Sagiv, Schwartz, 1995). Миграция может вступать в противоречие с ценностями сохранения, так как миграция часто приносит с собой изменение в привычный уклад жизни и социальные нормы, напротив, ценности открытости к изменениям предполагают ориентацию на получение нового опыта и впечатлений, чему и может способствовать общение с мигрантами, однако конкретно эта связь чисто теоретически может обосновываться неоднозначно ( $\mathrm{Da}$ vidov et al., 2008).

Ценности могут определеенным образом регулировать социальную дистанцию, т.е., другими словами, проницаемость границ между ин- и аутгруппой для обеспечения контакта, что в дальнейшем и может определять установки по отношению к миграции со стороны принимающего населения.

\section{Взаимосвязь ценностей, социальной дистанции и установок по отношению к миграции}

Можно предположить, что для представителей различных европейских культур существуют универсальные связи ценностей, социальной дистанции и установок по отношению к миграции. А именно ценности сохранения положительно связаны с социальной дистанцией по отношению к мигрантам, так как мигранты 
могут восприниматься в качестве угрозы безопасности, традициям, социальным нормам и правилам, сложившимся в принимающей стране, а ценности открытости изменениям отрицательно связаны с социальной дистанцией, так как эти ценности, прежде всего, предполагают ориентацию на новый опыт, новые переживания и перемены, а также фокус на индивида, а не на группу, саморазвитие, меньший уровень консерватизма, что, возможно, должно в большей мере обеспечивать подходящую основу для контактов с мигрантами.

В свою очередь, социальная дистанция положительно связана с ассоциацией миграции и негативных изменений в стране в социальной и экономической сфере, с мнением, что необходимо каким-либо способом если не запретить, то хотя бы ограничить миграцию, что естественным образом должно снизить вероятность контакта с представителями нежелательной группы в повседневной жизни. С другой стороны, отсутствие неприятия контакта с мигрантами может обеспечить возможность для распространения положительных установок по отношению к миграции.

Цель исследования. Целью настоящего исследования является проверка культурно-универсального характера связей между социальной дистанцией, ценностными ориентациями и установками по отношению к мигрантам.

Гипотезы исследования. Социальная дистанция положительно связана с ценностями сохранения и отрицательно с ценностями открытости к изменениям (Н1); социальная дистанция положительно связана с негативными установками по отношению к миграции (миграция как угроза и миграционный ценз) и отрицательно с положительными установками (желательность миграции) (Н2) - гипотезы отражены на рисунке 1; данный характер связей носит культурно-универсальный характер (Н3).

Гипотезы исследования

Рисунок 1

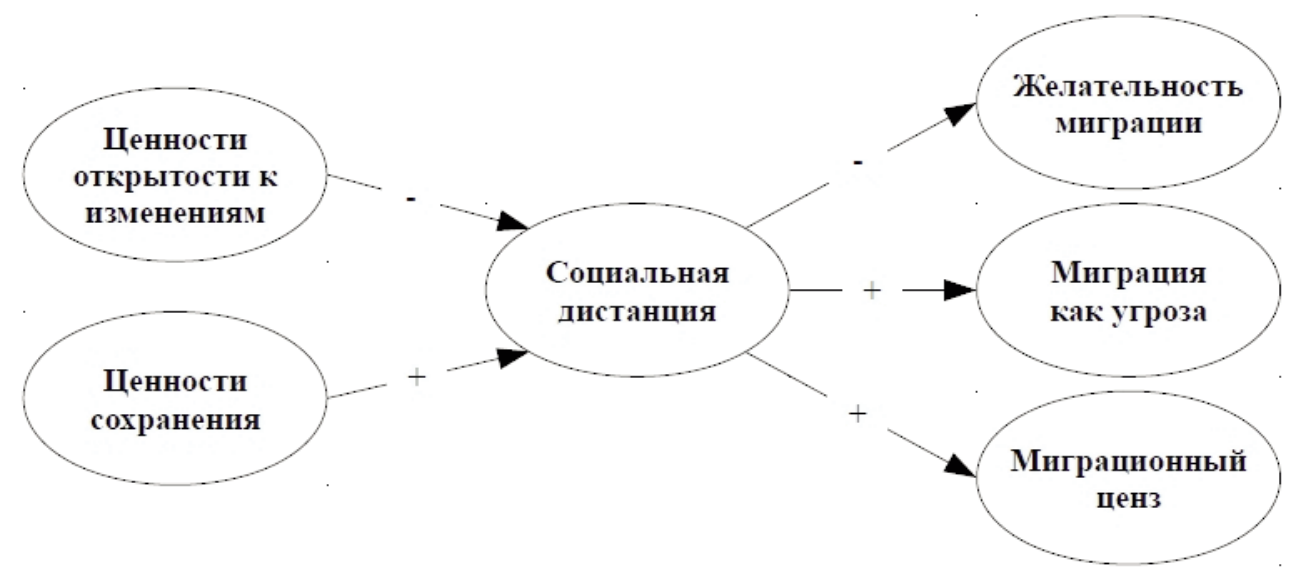




\section{Методика исследования}

Выборка. В статье используются данные 7-го раунда Европейского социального исследования за 2014 г. (ESS Round 7: European Social Survey Round 7 Data, 2014), были выбраны 4 страны-участницы этого раунда опроса, в которых проживает большое количество мигрантов, согласно данным статистической службы ЕС (Migration and Migrant Population Statistics, 2015), это Бельгия ( $\mathrm{N}=1618)$, Германия $(\mathrm{N}=2703)$, Франция $(\mathrm{N}=$ $=1650)$ и Нидерланды $(\mathrm{N}=1687)$, всего 7658 участников, представителей этнического большинства.

Инструментарий исследования. Ниже приводится описание шкал, использованных в исследовании, с примерным переводом вопросов на русский язык и кодами для поиска полной информации в документации Европейского социального исследования (см.: ESS Round 7: European Social Survey, 2015).

Ценностные ориентащии. Согласно теории ценностей Ш. Шварца (Schwartz, Butenko, 2014), были рассмотрены ценностные блоки Открытость к изменениям и Сохранение. Ценности открытости к изменениям, примеры вопросов: «Ему важно принимать свои собственные решения, касающиеся его жизни», «Ему важно испытывать разнообразные виды нового опыта» и т.д. (коды вопросов: He, Hg, Hn, Нp); Ценности сохранения, примеры вопросов: «Иметь сильное государство, которое может защитить своих граждан, очень важно для него», «Ему важно соблюдать правила, даже когда никто не наблюдает за ним» и т.д. (коды вопросов: $\mathrm{Hf}, \mathrm{Hj}, \mathrm{Hk}, \mathrm{Ho}$ ); ответы: 1 = Совсем не похож на меня, 6 = Очень похож на меня.

Социальная дистаниия. Респондентам предлагалось представить, что лица другой расы или этнической группы приезжают к ним в страну, и ответить, насколько для них приемлемо возникновение таких ситуаций в профессиональной и личностной сфере, в которых им предстоит какое-либо социальное взаимодействие с этими людьми. Примеры вопросов: «Насколько приемлемо, если такой человек был бы назначен вашим руководителем [на работе]?», «Насколько приемлемо, если такой человек вышел замуж/женился на вашем близком родственнике?» (коды вопросов: D10, D11); ответы: 0 = Полностью приемлемо, 10 = Полностью неприемлемо.

Желательность мигращии. В этом блоке вопросов респондентам предлагалось оценить, насколько позитивные изменения в экономической, культурной и социальной сфере связаны с миграцией из других стран. Здесь отражены базовые позитивные эффекты миграции, согласно идеологии мультикультурализма. Примеры вопросов: «Для экономики страны в целом хорошо или плохо, что люди приезжают сюда жить из других стран?», «С приездом мигрантов станет ли страна как место для жизни лучше или хуже?» (коды вопросов: В32, В33, В34); ответы: 0 = Плохо/хуже, 10 = Хорошо/лучше.

Миграция как угроза. В этом блоке вопросов респондентам предлагалось оценить негативные изменения в социальной и экономической сфере, связанные с миграцией из других стран: «миграция способствует повы- 
шению уровня преступности», «мигранты отнимают рабочие места», «мигранты забирают больше, чем отдают» - эти основные опасения, которые обычно высказываются в связи с проблемой миграции (Fitzgerald et al., 2012; Higgins et al., 2010; Simon, Sikich, 2007). Примеры вопросов: «С приездом мигрантов улучшилась или ухудшилась ситуация с рабочими местами в стране?», «С приездом мигрантов улучшилась или ухудшилась ситуация с преступностью в стране?» (коды вопросов: D7, D8, D9); ответы: 0 = Улучшилась, 10 = Ухудшилась.

Миграционный ценз. В этом блоке вопросов респондентам предлагалось оценить, насколько для них важно, чтобы приезжие соответствовали определенным ограничительным условиям, будь то уровень образования или языковые навыки, примеры вопросов: «Насколько важно иметь хорошее образование?», «Насколько важно уметь разговаривать на государственном языке?» (коды вопросов: D1, D2, D5); ответы: $0=$ Совсем не важно, 10 = Очень важно.

Социально-демографические переменные. Кроме пола и возраста, учитывалась также степень религиозности респондентов, которая оценивалась как прямым вопросом «Насколько вы религиозны?», так и вопросами касающимися вовлеченности в религиозное поведение, примеры вопросов: «Как часто вы посещаете религиозные службы, кроме особых случаев?», «За исключением участия в богослужениях, как часто вы молитесь?» (коды вопросов: С13, C14, С15); ответы: 1 = Никогда, 7 = Каждый день.

\section{Результаты}

Для проверки факторной структуры использованных шкал, а также их метрической инвариантности с помощью программы AMOS были проведены конфирматорный факторный анализ (CFA) и мультигрупповой конфирматорный факторный анализ (MG-CFA) ${ }^{1}$, результаты отражены в таблице 1.

Показатели моделей по странам и измерительная модель (MG-CFA) соответствуют рекомендуемым: CFI > .90; TLI > .90; RMSEA < .06; SRMR <.08 (Hu, Bentler, 1999; Hair et al., 2010) для того, чтобы заключить о соответствии моделей эмпирическим данным² $^{2}$ Для определения метрической инвариантности были рассмотрены следующиерекомендации CFI $\leqslant .010$; TLI $\leqslant .010 ;$ RMSEA $\leqslant .015 ;$ SRMR $\leqslant .030$ (Chen, 2007), согласно которым можно говорить о полной метрической инвариантности (сильной факторной инвариантности), что является достаточным для рассмотрения связей между конструктами в межстрановом сравнении (Vandenberg, Lance, 2000).

Далее были рассмотрены показатели надежности, конвергентной и дискриминантной валидности шкал.

\footnotetext{
${ }^{1}$ Для расчетов использовались корреляционные матрицы со взвешенными наблюдениями.

${ }^{2}$ Здесь и далее показатели по $\chi^{2}$ игнорируются в связи с большим объемом выборки и количеством наблюдаемых переменных, так как в таком случае данный критерий становится неадекватным для оценки моделей (см.: Hair et al., 2010; Milfont, Fischer, 2010).
} 
Показатели пригодности моделей

\begin{tabular}{|c|c|c|c|c|c|c|c|c|c|c|}
\hline & $\chi^{2}$ & df & $\begin{array}{l}\text { RMSEA } \\
\text { [CI 90\%] }\end{array}$ & RMSEA & SRMR & SRMR & CFI & CFI & TLI & $\triangle$ TLI \\
\hline \multicolumn{11}{|c|}{ Модели по странам } \\
\hline Бельгия & $672.799^{*}$ & 188 & $\begin{array}{c}.040 \\
{[.037, .037]}\end{array}$ & & .0391 & & .949 & & .937 & \\
\hline Германия & $1188.166^{*}$ & 188 & $\begin{array}{c}.045 \\
{[.042, .047]}\end{array}$ & & .0373 & & .949 & & .937 & \\
\hline Франция & $884.800^{*}$ & 188 & $\begin{array}{c}.047 \\
{[.044, .050]}\end{array}$ & & .0426 & & .938 & & .923 & \\
\hline Нидерланды & $836.393^{*}$ & 188 & $\begin{array}{c}.045 \\
{[.042, .049]} \\
\end{array}$ & & .0468 & & .940 & & .927 & \\
\hline \multicolumn{11}{|c|}{ Измерительная модель } \\
\hline $\begin{array}{l}\text { Конфигураль- } \\
\text { ная }\end{array}$ & $3582.170^{*}$ & 752 & $\begin{array}{c}.022 \\
{[.022, .023]}\end{array}$ & & .0373 & & .945 & & .932 & \\
\hline Метрическая & $4043.677^{*}$ & 797 & $\begin{array}{c}.023 \\
{[.022, .024]}\end{array}$ & .001 & .0392 & .0019 & .936 & .009 & .926 & .006 \\
\hline \multicolumn{11}{|c|}{ Структурная модель } \\
\hline $\begin{array}{l}\text { Конфигураль- } \\
\text { ная }\end{array}$ & $5243.969^{*}$ & 912 & $\begin{array}{c}.025 \\
{[.024, .026]}\end{array}$ & & .0451 & & .920 & & .903 & \\
\hline Метрическая & $5701.827^{*}$ & 957 & $\begin{array}{c}.026 \\
{[.025, .026]}\end{array}$ & .001 & .0466 & .0015 & .912 & .008 & .898 & .005 \\
\hline
\end{tabular}

Примечание. $\chi^{2}$ - значение статистики хи-квадрат; $\mathrm{df}$ - число степеней свободы; RMSEA корень среднеквадратической ошибки аппроксимации; CI 90\% - границы доверительного интервала для RMSEA; SRMR - стандартизированный корень среднеквадратического остатка; CFI - сравнительный индекс соответствия Бентлера; TLI - индекс Такера-Льюиса.

$*-p<.001$.

Были рассчитаны показатель составной надежности $\rho$-Рейкова, показатель средней извлеченной дисперсии (AVE), показатель максимальной разделенной дисперсии (MSV), показатель средней разделенной дисперсии (ASV), для проверки дискриминантной валидности дополнительно был проведен тест ФорнеллаЛаркера. Результаты всех тестов отражены в таблице 2.

Показатели должны быть следующими: шкала надежна, если CR > .70; конвергентная валидность есть, если
$\mathrm{AVE}>.50$; дискриминантная валидность есть, если MSV < AVE, ASV < AVE (Hair et al., 2010), а также если квадратный корень из средней извлеченной дисперсии (AVE) больше, чем коэффициенты корреляции между конструктами (Fornell, Lacker, 1981). Итак, можно отметить удовлетворительные показатели составной надежности и коэффициенты $\alpha$ Кронбаха для всех шкал. Проблемы с конвергентной валидностью наблюдаются у обеих шкал ценностных ориентаций, что в принципе характерно для 


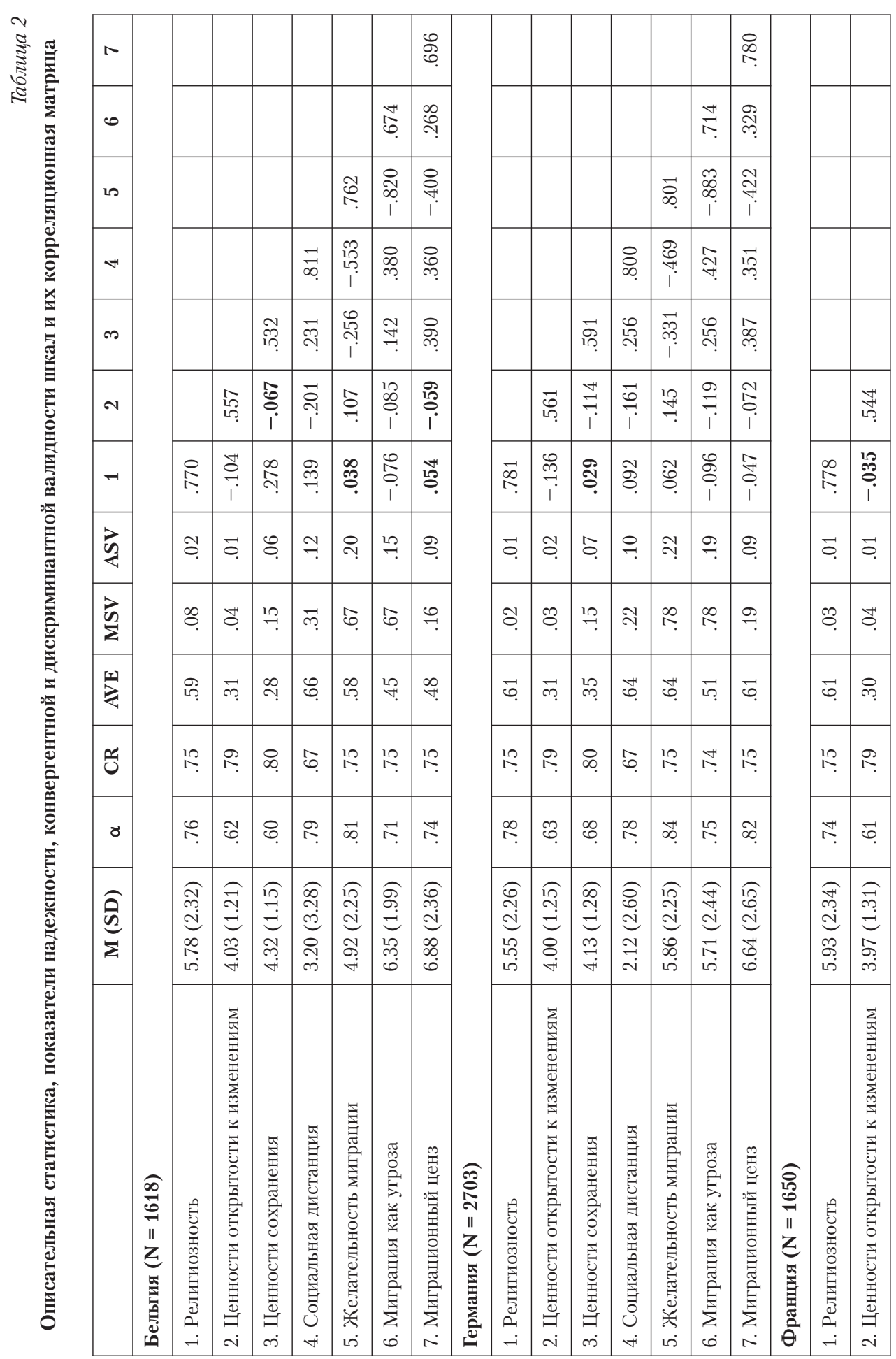




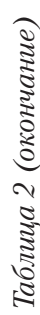

\begin{tabular}{|c|c|c|c|c|c|c|c|c|c|c|c|c|c|}
\hline N & & & & & $\stackrel{ }{N}$ & & & & & & & & $\vec{N}$ \\
\hline 0 & & & & $\vec{\infty}$ & $\stackrel{\text { ঙ }}{\text { ণ }}$ & & & & & & & \& & 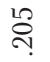 \\
\hline 18 & & & $\stackrel{\overbrace{}}{\infty}$ & 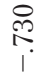 & 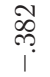 & & & & & & సิ & $\begin{array}{l}\infty \\
\infty \\
\infty \\
1 .\end{array}$ & 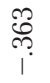 \\
\hline$\checkmark$ & & ๓ి & 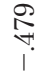 & ๗ิ & $\begin{array}{l}\text { กิ } \\
\text { กิ }\end{array}$ & & & & & ஜ̊ & $\underset{\uparrow}{\stackrel{+}{?}}$ & ले & $\frac{\circ}{\text { m. }}$ \\
\hline מח & $\begin{array}{l}\mathscr{R} \\
\text { in }\end{array}$ & $\stackrel{\rightleftarrows}{\stackrel{\Perp}{*}}$ & ஓे & $\stackrel{\Re}{=}$ & 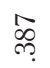 & & & & ஓ & 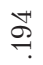 & 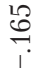 & $\vec{\sigma}$ & $\begin{array}{l}\text { ฟै } \\
\text { }\end{array}$ \\
\hline N & ๙ิ & $\underset{1}{\stackrel{8}{\rightleftarrows}}$ & 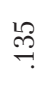 & Ðे & 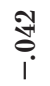 & & & శో & $\begin{array}{l}\infty \\
\stackrel{0}{0} \\
1\end{array}$ & $\stackrel{\bar{\sigma}}{\rightleftarrows}$ & $\ddot{8}$ & 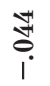 & 농 \\
\hline- & $\stackrel{\infty}{\longrightarrow}$ & $\begin{array}{l}\infty \\
\infty \\
0\end{array}$ & ֻั. & శ్ & $\stackrel{0}{\stackrel{0}{0}}$ & & ठิ & $\stackrel{\curvearrowright}{\stackrel{I}{\longrightarrow}}$ & ๗ิ & ث্ & ปิ & $\stackrel{\nabla}{0}$ & $\stackrel{0}{0}$ \\
\hline$\frac{3}{4}$ & so. & $\stackrel{\infty}{\circ}$. & $\stackrel{\rightleftharpoons}{\rightleftharpoons}$ & $\stackrel{?}{?}$ & $\stackrel{\infty}{\circ}$ & & ชิ & 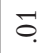 & $\ddot{0}$ & $\stackrel{8}{0}$ & શิ & $\stackrel{0}{\bullet}$ & 능 \\
\hline$\vec{n}$ & $\stackrel{12}{\rightarrow}$ & ๆิ & กึ? & กึ? & $\stackrel{10}{\rightarrow}$ & & ㄴ? & ஜ & $\stackrel{2}{0}$ & 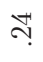 & $\stackrel{R}{R}$ & $\stackrel{R}{T}$ & $\stackrel{m}{\rightarrow}$ \\
\hline${ }_{2}^{n}$ & શి & O̊. & $\vec{T}$ & $\stackrel{\leftrightarrow}{*}$ & ำ & & ठ઼. & ก? & กึ & $\theta^{-3}$ & กิ & $\stackrel{乛}{\underset{?}{+}}$ & กิ \\
\hline گૈ & $\infty$ & 6 & $\stackrel{12}{\stackrel{1}{r} . ~}$ & $\stackrel{\sim}{r}$ & $\stackrel{2}{\sim}$ & & $\stackrel{2}{?}$ & $\stackrel{P}{\Gamma}$ & $\infty$ & $\widehat{0}$ & $\stackrel{10}{\uparrow}$ & $\stackrel{10}{\Gamma}$ & $\stackrel{12}{\Gamma}$ \\
\hline ช & $\tilde{\sigma}$ & నం & œ & F & $\stackrel{N}{r}$ & & $\stackrel{P}{P}$ & ?อ & $\widehat{6}$ & $\stackrel{\infty}{?}$ & $\curvearrowright$ & $\stackrel{10}{P}$ & $\stackrel{12}{?}$ \\
\hline$\underset{\Sigma}{\hat{\sigma}}$ & 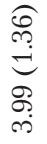 & 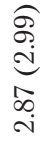 & 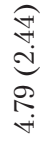 & 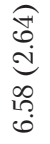 & 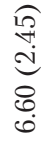 & & 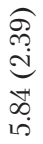 & 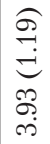 & 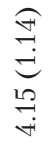 & 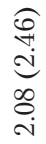 & 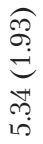 & 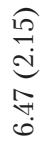 & 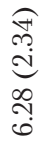 \\
\hline & 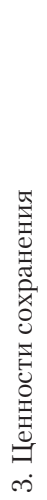 & 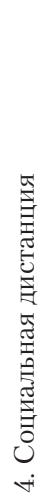 & 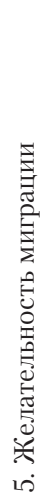 & 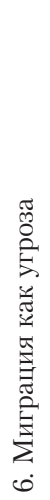 & 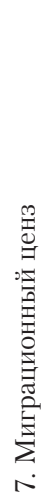 & 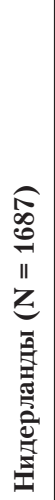 & 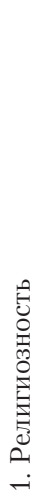 & 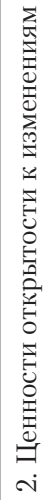 & 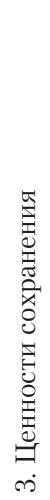 & 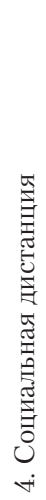 & 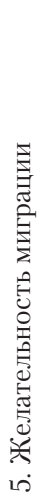 & 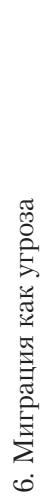 & 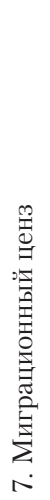 \\
\hline
\end{tabular}

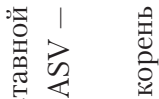

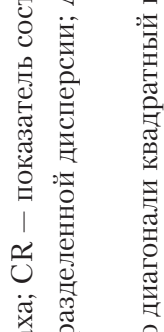

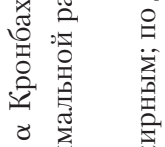

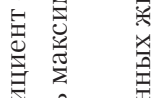

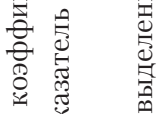

1 응

ช

密

: $V$

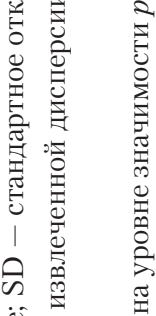

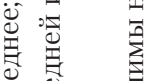

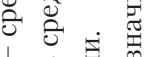

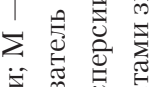

噉

잉

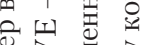

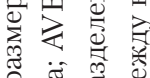

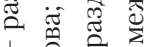

乙啳啄

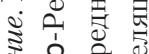

횔

है

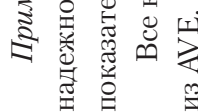


самой методики Ш. Шварца (см.: Schwartz et al., 2012; Davidov, Meuleman, 2012). Также неудовлетворительными являются показатели дискриминантной валидности для шкал Желательность миграции и Миграция как угроза; можно считать, что это один конструкт и что вопросы одной шкалы являются просто обратными к другой.

Однако показатели AVE являются очень строгим критерием, поэтому в первом случае при удовлетворительном показателе составной надежности (CR), несмотря на то, что более $50 \%$ дисперсии объясняется ошибкой измерения, шкала может быть пригодна для дальнейшего анализа (Malhotra, Dash, 2010), что касается шкалы Желательность миграции и Миграция как угроза, то из концептуальных соображений, исходя из содержательной стороны вопросов шкалы, их можно считать отличимыми.
Таким образом, можно перейти непосредственно к проверке гипотез исследования.

Для проверки гипотез исследования было проведено моделирование структурными уравнениями (SEM), показатели пригодности модели отражены в таблице 1, а оценки регрессионных связей - в таблице 3 и на путевой диаграмме (рисунок 2).

Результаты анализа подтверждают все три гипотезы исследования (для удобства данные связи отдельно отображены на рисунке 3 ). Однако размеры эффекта для некоторых зависимых переменных (см. таблицу 4) указывают, что выбор некоторых объясняющих переменных в модели не является вполне удовлетворительным. Также, помимо этих универсальных связей, можно говорить о том, что многие другие связи в модели носят культурно-специфический характер, особенно примечательным

Рисунок 2

\section{Путевая диаграмма}

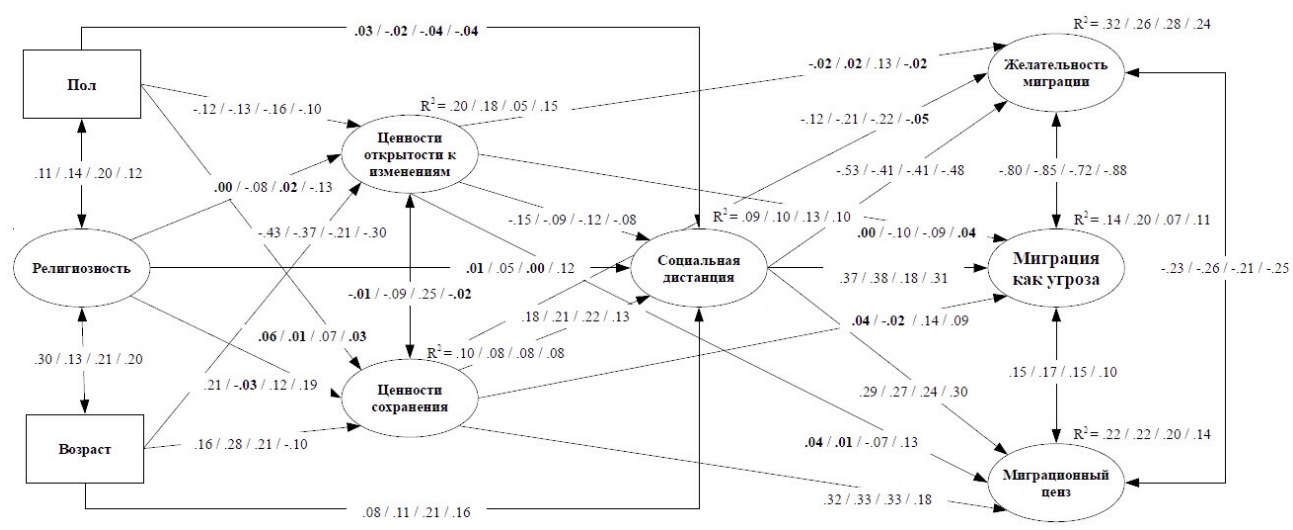

Примечание. Стандартизированные регрессионные коэффициенты, по порядку: Бельгия, Германия, Франция, Нидерланды. Все регрессионные коэффициенты значимы на уровне значимости $p<.05$, кроме выделенных полужирным шрифтом. 


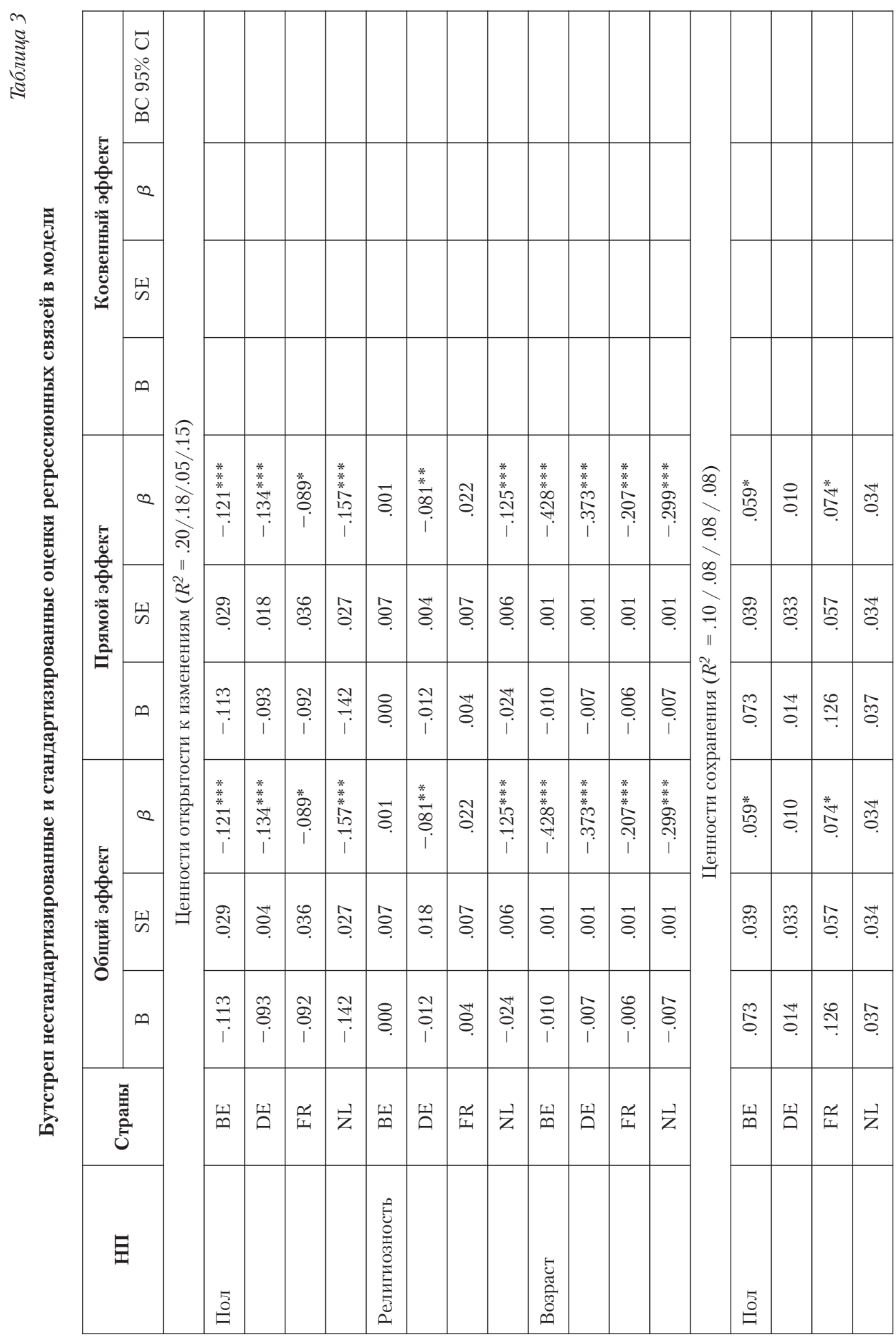




\begin{tabular}{|c|c|c|c|c|c|c|c|c|c|c|c|c|c|c|c|c|c|c|c|c|}
\hline \multirow{4}{*}{ 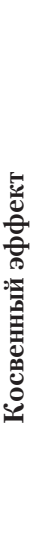 } & 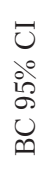 & & & & & & & & & \multirow{12}{*}{ 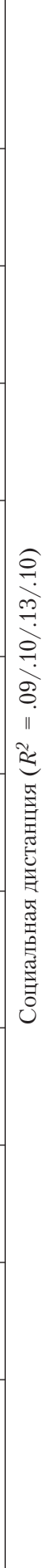 } & 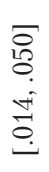 & 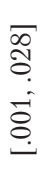 & $\begin{array}{c}0 \\
0 \\
0 \\
0 \\
-1 \\
0 \\
0 \\
0\end{array}$ & 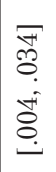 & 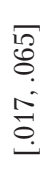 & $\begin{array}{l}\bar{\sigma} \\
0 \\
0 \\
0 \\
0 \\
i\end{array}$ & $\begin{array}{l}\overline{2} \\
\stackrel{2}{8} \\
0 \\
0 \\
0 \\
0 \\
0\end{array}$ & 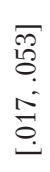 & $\begin{array}{l}\sqrt{2} \\
\stackrel{9}{\rightarrow} \\
\stackrel{0}{0} \\
\stackrel{8}{0}\end{array}$ & 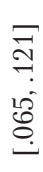 \\
\hline & $Q$ & & & & & & & & & & $\begin{array}{l}\stackrel{*}{*} \\
\stackrel{*}{*} \\
\stackrel{\text { İ }}{0}\end{array}$ & $\stackrel{\stackrel{*}{*}}{\sigma}$ & $\begin{array}{l}\stackrel{*}{*} \\
\stackrel{*}{\sigma} \\
\text {. }\end{array}$ & $\stackrel{\stackrel{*}{*}}{\stackrel{*}{\sigma}}$ & $\begin{array}{l}* \\
* \\
* \\
\infty \\
\cdots \\
0\end{array}$ & ฮิ & 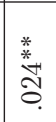 & $\begin{array}{l}\stackrel{*}{*} \\
\stackrel{*}{*} \\
\stackrel{0}{0}\end{array}$ & $\begin{array}{l}\stackrel{*}{*} \\
\stackrel{*}{*} \\
\stackrel{2}{\delta}\end{array}$ & $\stackrel{\text { * }}{*}$ \\
\hline & 崩 & & & & & & & & & & Ðે & 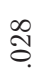 & $\stackrel{2}{8}$ & จิ & $\stackrel{m}{0}$ & \&̊. & $\stackrel{\infty}{8}$ & $\hat{8}$ & $\ddot{8}$ & ฮै \\
\hline & $\varphi$ & & & & & & & & & & 声 & 농 & 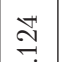 & $\hat{\theta}$ & $\stackrel{ }{\stackrel{\circ}{0}}$ & §ิ & ন্ర & $\stackrel{\infty}{\stackrel{0}{0}}$ & $\stackrel{m}{\sigma}$ & $\stackrel{\circ}{0}$ \\
\hline \multirow{3}{*}{ 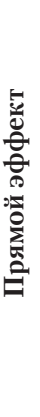 } & $Q$ & $\begin{array}{l}\stackrel{*}{*} \\
\stackrel{*}{*} \\
\stackrel{2}{2}\end{array}$ & 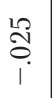 & $\begin{array}{l}\stackrel{*}{*} \\
\stackrel{*}{g} \\
\exists\end{array}$ & \begin{tabular}{l}
$\frac{*}{*}$ \\
$\stackrel{*}{*} 0$ \\
$\infty$ \\
\hdashline
\end{tabular} & $\begin{array}{l}\stackrel{*}{*} \\
\stackrel{*}{*} \\
\stackrel{6}{\bullet}\end{array}$ & 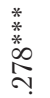 & $\begin{array}{l}* \\
* \\
* \\
\stackrel{*}{*} \\
\stackrel{*}{*}\end{array}$ & $\stackrel{*}{\stackrel{*}{*}}$ & & ஜి & $\overrightarrow{\tilde{O}}$ & 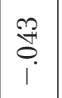 & 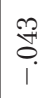 & $\stackrel{\Delta}{0}$ & $\stackrel{\infty}{\stackrel{\infty}{0} . ~}$ & §. & $\stackrel{\text { * }}{\stackrel{*}{\rightleftharpoons}}$ & $\begin{array}{l}\stackrel{*}{*} \\
\mathscr{0} \\
0\end{array}$ & $\stackrel{\stackrel{*}{*}}{\stackrel{*}{\leftrightarrows}}$ \\
\hline & II & $\ddot{8}$ & s. & $\stackrel{0}{0}$ & $\stackrel{\infty}{8}$ & $\overline{8}$ & $\overline{8}$ & ڤิ & $\overline{8}$ & & $\stackrel{\Re}{\underset{十}{+}}$ & $\bar{g}$ & $\stackrel{\widehat{m}}{\rightarrow}$ & $\stackrel{\leftrightarrow}{\rightleftarrows}$ & 梁 & ̊ั. & ஜి & $\begin{array}{l}\text { ڤै } \\
\text { ô. }\end{array}$ & $\stackrel{2}{8}$ & $\stackrel{\circledast}{8}$ \\
\hline & $\infty$ & ปิ & 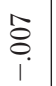 & ஜి & $\stackrel{\Im}{0}$ & $\stackrel{2}{8}$ & $\stackrel{\circ}{\circ}$ & $\stackrel{0}{0}$ & $\stackrel{8}{\circ}$ & & $\stackrel{1}{\stackrel{2}{น}}$ & $\begin{array}{l}0 \\
\infty \\
0 \\
1\end{array}$ & $\stackrel{\stackrel{\Im}{\circ}}{\longrightarrow}$ & 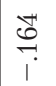 & $\stackrel{\Delta}{0}$ & $\stackrel{ }{\stackrel{1}{0}}$ & \&. & $\stackrel{2}{\mathscr{2}}$ & $\vec{\sigma}$ & อี. \\
\hline \multirow{3}{*}{ 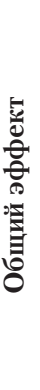 } & $Q$ & 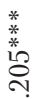 & $\begin{array}{l}\stackrel{2}{0} \\
\stackrel{0}{0} \\
\text { । }\end{array}$ & $\begin{array}{l}\stackrel{*}{*} \\
\stackrel{*}{g} \\
=\end{array}$ & $\begin{array}{l}\stackrel{*}{*} \\
\stackrel{*}{*} \\
\stackrel{0}{\infty} \\
\stackrel{0}{\sim}\end{array}$ & $\begin{array}{l}\stackrel{*}{*} \\
\stackrel{*}{*} \\
\stackrel{6}{\sim}\end{array}$ & 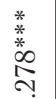 & $\begin{array}{l}* \\
\stackrel{*}{*} \\
\stackrel{*}{*} \\
\stackrel{2}{\ominus}\end{array}$ & $\begin{array}{l}\stackrel{*}{*} \\
\stackrel{*}{*} \\
\stackrel{\infty}{\sim}\end{array}$ & & 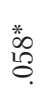 & $\stackrel{0}{0}$ & $\stackrel{0}{0}$ & \&ै. & 농 & 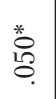 & \ّ & $\stackrel{*}{*}$ & $\begin{array}{l}\stackrel{*}{*} \\
\stackrel{*}{*} \\
\stackrel{2}{ }\end{array}$ & $\begin{array}{l}\stackrel{*}{*} \\
\stackrel{*}{*} \\
\stackrel{\sim}{\text { ? }}\end{array}$ \\
\hline & 닝 & 8 & so & $\stackrel{\Xi}{o}$ & $\stackrel{\infty}{8}$ & $\overline{8}$ & $\overline{8}$ & §ิ & $\overline{8}$ & & $\underset{ت}{\rightleftarrows}$ & \& & $\stackrel{m}{\rightarrow}$ & $\stackrel{?}{\stackrel{2}{\circ}}$ & 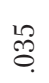 & §ิ & ঐి & $\stackrel{\text { ำ }}{0}$ & ठे & ชิ \\
\hline & 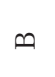 & ำ & $\hat{8}$ & ஜे. & $\stackrel{\mathscr{P}}{0}$ & $\stackrel{2}{\stackrel{8}{8}}$ & $\stackrel{\circ}{\circ}$ & $\stackrel{\circ}{\circ}$ & $\stackrel{2}{8}$ & & ஓ्: & $\stackrel{\overbrace{}}{\stackrel{\Xi}{0}}$ & $\stackrel{m}{5}$ & $\stackrel{\infty}{\mathscr{8}}$ & ל艹 & ঙี & ิิ & $\stackrel{\Re}{\neg}$ & ठ艹 & సิ \\
\hline \multicolumn{2}{|c|}{ 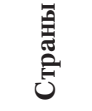 } & $\stackrel{\text { II }}{\infty}$ & 峼 & $\frac{\not c}{1 .}$ & $\overrightarrow{\mathrm{z}}$ & 됨 & $\stackrel{\text { II }}{\circ}$ & $\frac{\alpha}{I I}$ & $\vec{z}$ & & $\stackrel{1}{D}$ & 㭊 & $\frac{q}{I I}$ & $\vec{z}$ & 됨 & 㞬 & $\frac{\not c L}{I I}$ & $\vec{z}$ & 딤 & 嘼 \\
\hline \multicolumn{2}{|c|}{ 亚 } & \multicolumn{4}{|c|}{ 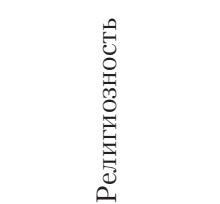 } & \multicolumn{4}{|c|}{ 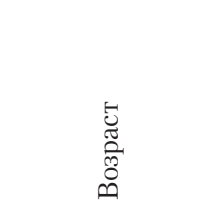 } & & \multicolumn{4}{|c|}{$\stackrel{5}{\ominus}$} & \multicolumn{4}{|c|}{ 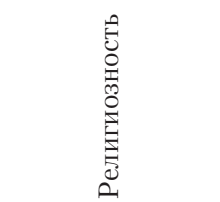 } & . & 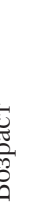 \\
\hline
\end{tabular}




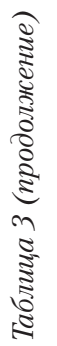

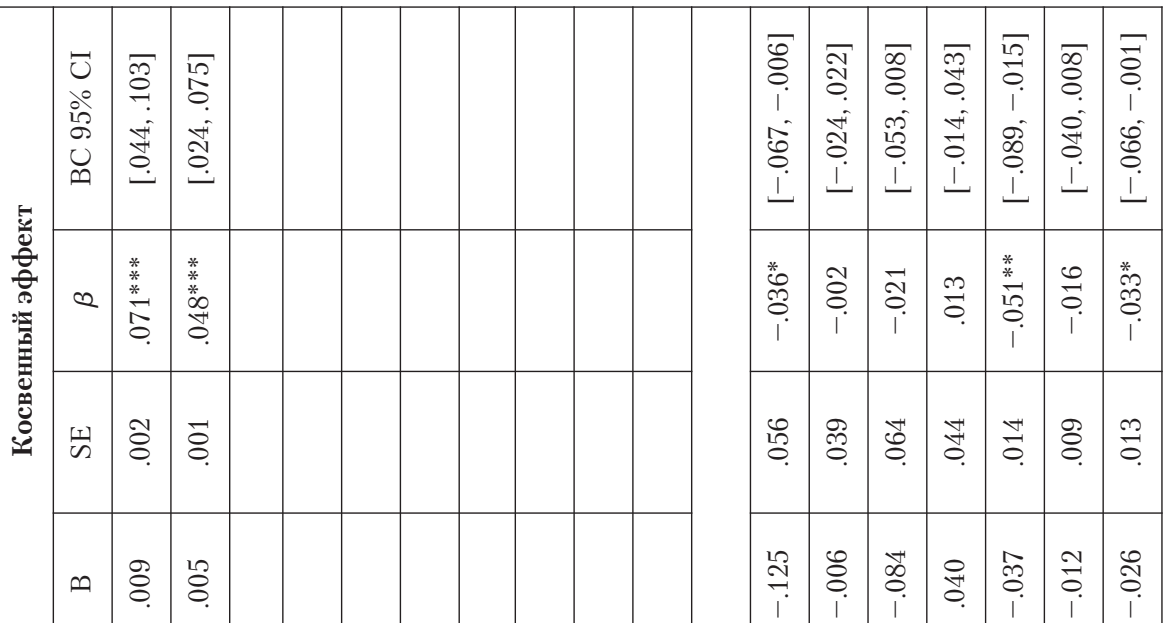

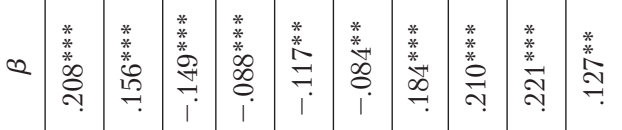

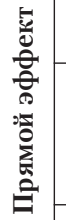

ঙ্ฺ

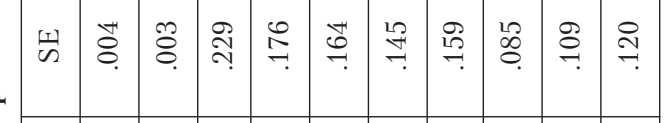

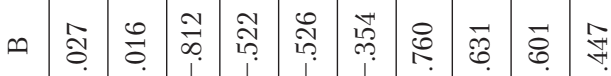

Q

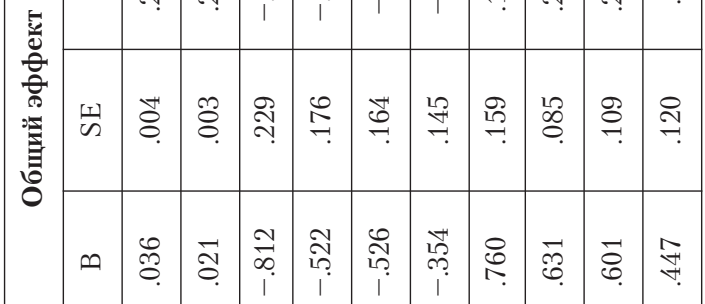

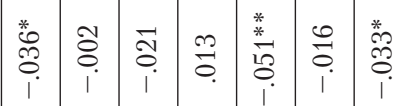

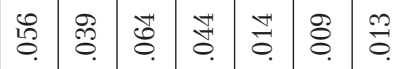

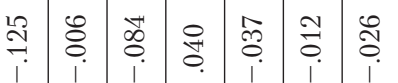

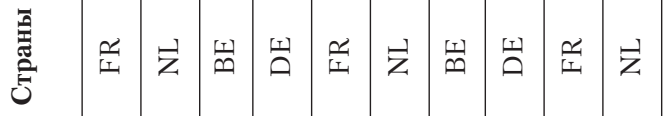

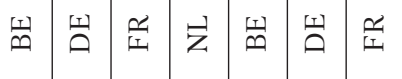

三

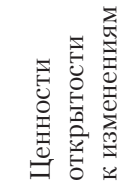

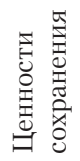

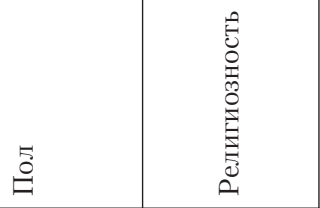




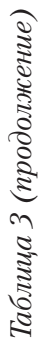

\begin{tabular}{|c|c|c|c|c|c|c|c|c|c|c|c|c|c|c|c|c|c|c|}
\hline \multirow{4}{*}{ 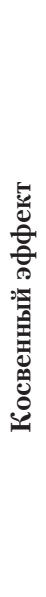 } & $\begin{array}{l}\text { U } \\
\stackrel{0}{ } \\
\stackrel{\sigma}{O} \\
ن\end{array}$ & 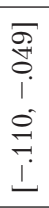 & $\begin{array}{l}\overline{8} \\
0 \\
1 \\
1 \\
\stackrel{4}{7} \\
\stackrel{1}{1}\end{array}$ & 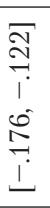 & 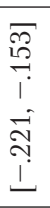 & 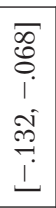 & 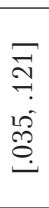 & 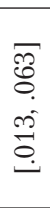 & 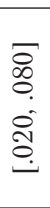 & $\begin{array}{l}\text { कू } \\
\text { 今. } \\
\dot{0} \\
\dot{0}\end{array}$ & 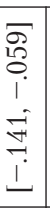 & \begin{tabular}{l}
$\bar{\nabla}$ \\
0 \\
1 \\
$\hat{0}$ \\
\hdashline \\
1
\end{tabular} & 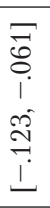 & 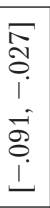 & & & & \\
\hline & Q & 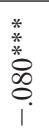 & $\underset{\text { । }}{\stackrel{*}{*}}$ & $\underset{\mid}{\stackrel{*}{*}} \stackrel{\stackrel{*}{*}}{\stackrel{*}{*}}$ & 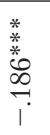 & 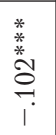 & 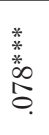 & 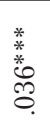 & $\begin{array}{l}\text { * } \\
\stackrel{0}{0} \\
\stackrel{0}{0} \\
\stackrel{0}{0}\end{array}$ & $\stackrel{*}{\stackrel{*}{+}}$ & 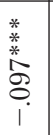 & $\begin{array}{l}\text { * } \\
* \\
\text { * }\end{array}$ & $\begin{array}{l}\stackrel{*}{*} \\
\stackrel{*}{8} \\
\stackrel{*}{\circ}\end{array}$ & $\begin{array}{l}* \\
* \\
* \\
\stackrel{*}{*} \\
\stackrel{0}{0}\end{array}$ & & & & \\
\hline & 닝 & $\stackrel{0}{0}$ & $\stackrel{\text { }}{8}$ & $\overline{8}$ & ฮै. & $\overline{8}$ & $\begin{array}{l}10 \\
0 \\
0\end{array}$ & ஜै & $\bar{\phi}$ & ڤ̊ & $\overrightarrow{8}$ & $\overrightarrow{8}$ & $\vec{\Delta}$ & ङે & & & & \\
\hline & 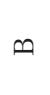 & $\stackrel{\overrightarrow{8}}{\overrightarrow{0}}$ & $\stackrel{0}{0}$ & $\underset{i}{\Delta}$ & 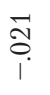 & $\stackrel{\infty}{0}$ & ๙ึ & $\underset{-\infty}{\infty}$ & $\stackrel{\infty}{\stackrel{\infty}{-}}$ & $\stackrel{m}{\leftrightarrow}$ & 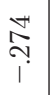 & $\stackrel{\Gamma}{\stackrel{\Gamma}{i}}$ & $\stackrel{10}{\stackrel{2}{1}}$ & $\stackrel{\infty}{\stackrel{\infty}{1}}$ & & & & \\
\hline \multirow{3}{*}{ 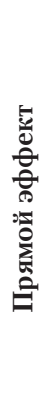 } & Q & & & & & & $\stackrel{0}{0}$ & $\frac{a}{\sigma}$. & $\begin{array}{l}\stackrel{*}{*} \\
\stackrel{*}{*} \stackrel{\text { I }}{\sim}\end{array}$ & $\stackrel{5}{0}$ & 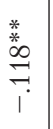 & $\underset{\stackrel{*}{*}}{\stackrel{*}{*}}$ & $\stackrel{\stackrel{*}{*} \stackrel{*}{*}}{\stackrel{*}{\sim}}$ & : & 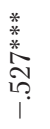 & 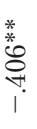 & 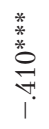 & 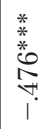 \\
\hline & 넨 & & & & & & 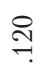 & $\stackrel{\mathscr{I}}{\stackrel{2}{*}}$ & 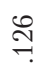 & $\stackrel{\circ}{\circ}$ & $\stackrel{0}{\circ}$ & $\stackrel{\infty}{\mathscr{\ell}}$ & कo & $\stackrel{8}{8}$ & ֻึ & 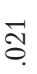 & 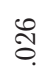 & ָิ \\
\hline & 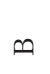 & & & & & & 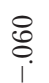 & ১ో & ஜै & $\begin{array}{l}\infty \\
\stackrel{2}{0}\end{array}$ & 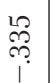 & 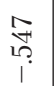 & $\overrightarrow{\vec{r}}$ & 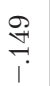 & $\begin{array}{l}\overrightarrow{0} \\
\text { ஜి }\end{array}$ & ָै & $\begin{array}{l}\infty \\
\text { مִ } \\
\text { m. }\end{array}$ & ని \\
\hline
\end{tabular}

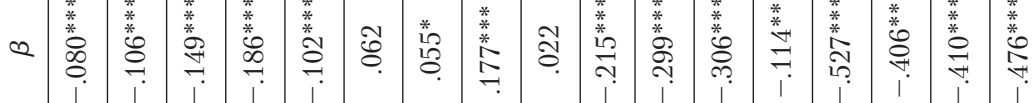

סै:

\begin{tabular}{|c|c|c|c|c|c|c|c|c|c|c|c|c|c|c|c|c|c|}
\hline 帘 & $\stackrel{0}{\circ}$ & ชิ & $\overline{8}$ & ชิ & $\overline{8}$ & ڤ్ & $\stackrel{\mathscr{m}}{\longrightarrow}$ & $\stackrel{\infty}{\stackrel{\infty}{\rightarrow}}$ & $\stackrel{\infty}{=}$ & 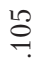 & ठ̊. & S. & $\stackrel{8}{-}$ & ๗ิ & 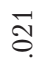 & $\begin{array}{l}\text { Iै } \\
\text { o. }\end{array}$ & ồ \\
\hline 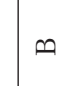 & 롱. & $\stackrel{0}{0}$ & $\underset{i}{\stackrel{H}{0}}$ & $\begin{array}{l}\vec{\sigma} \\
\text { ।. }\end{array}$ & $\stackrel{\infty}{8}$ & थึ & $\underset{\text { స̦ }}{\stackrel{\Delta}{n}}$ & $\vec{\delta}$ & ln & $\begin{array}{l}8 \\
\text { o. }\end{array}$ & $\underset{1}{\stackrel{Ð}{~}}$ & $\underset{i}{\stackrel{N}{N}}$ & $\stackrel{\vec{m}}{i}$ & $\begin{array}{l}\overrightarrow{0} \\
\text { p. }\end{array}$ & 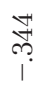 & 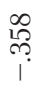 & 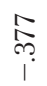 \\
\hline 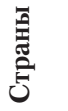 & $\dot{\mathrm{Z}}$ & 됨 & 贸 & $\stackrel{q u}{\underline{I}}$ & $\vec{z}$ & 뎀 & 넴 & 寽 & $\overrightarrow{\mathrm{z}}$ & 됨 & 됨 & $\frac{q u}{I}$ & $\vec{z}$ & $\frac{1}{\infty}$ & 劉 & 寽 & $\vec{z}$ \\
\hline 正 & 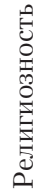 & & 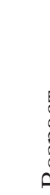 & & & & 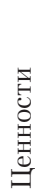 & 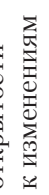 & & & & & & & 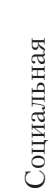 & & \\
\hline
\end{tabular}




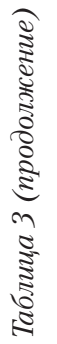

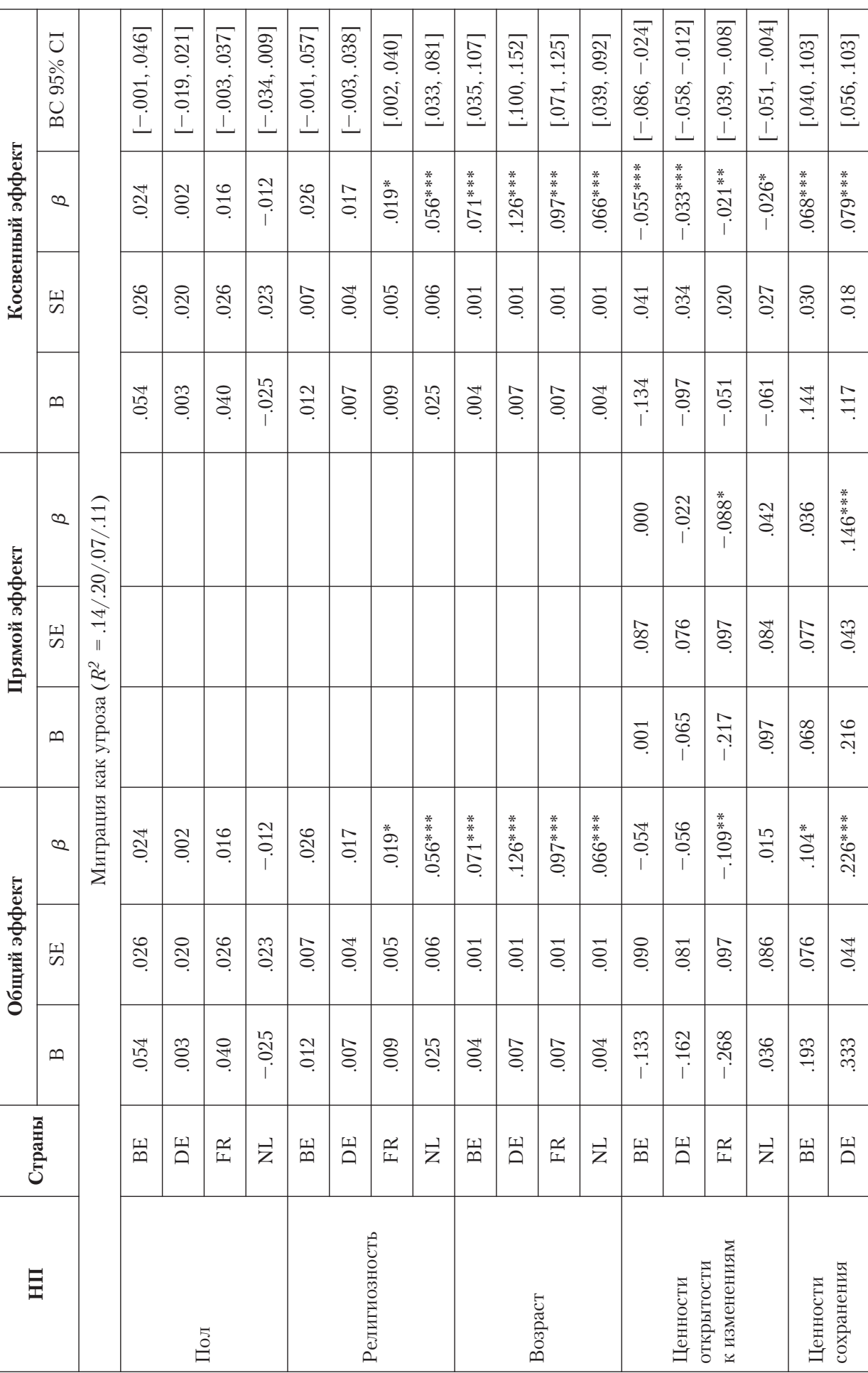




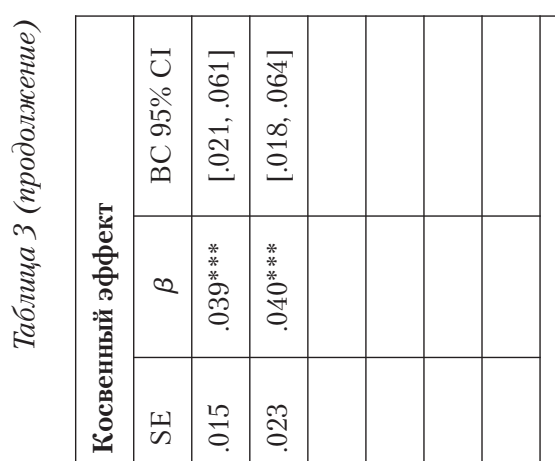

\begin{tabular}{|c|c|c|c|c|c|c|c|c|c|c|c|}
\hline & & & & & & & & & & & \\
\hline 㦿 & 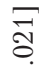 & $\begin{array}{l}\sqrt{2} \\
\text { है }\end{array}$ & $\overline{8}$ & $\stackrel{5}{\Xi}$ & $\begin{array}{l}\bar{\infty} \\
\stackrel{0}{0}\end{array}$ & $\begin{array}{l}\bar{\Omega} \\
5\end{array}$ & $\stackrel{8}{8}$ & $\begin{array}{l}\infty \\
\stackrel{\infty}{\sim}\end{array}$ & 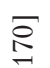 & $\underset{\infty}{\infty}$ & శ్ \\
\hline हो & ชิ & $\dot{8}$ & $\stackrel{150}{0}$ & $\dot{8}$ & $\stackrel{\infty}{0}$ & $\frac{10}{8}$ & $\hat{m}$ & $\stackrel{\leftrightarrow}{\stackrel{7}{0}}$ & $\stackrel{\dot{0}}{=}$ & $\stackrel{\vdots}{\Xi}$ & \\
\hline 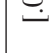 & $1^{\circ}$ & 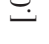 & $\perp^{\circ}$ & - & I & تَك & - & . & 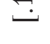 & & \\
\hline & 8 & ث: & సิ & 晜 & ㄴㅇㅇ & 裉 & 蒾 & 莡 & 䊪 & 菜 & \\
\hline & & $v$ & & $\stackrel{8}{0}$ & 0 & $\stackrel{?}{0}$ & $\vec{\varnothing}$ & $\stackrel{1}{\infty}$ & $\stackrel{\Re}{\stackrel{?}{\rightarrow}}$ & $\stackrel{\vec{\Omega}}{\sim}$ & \\
\hline
\end{tabular}

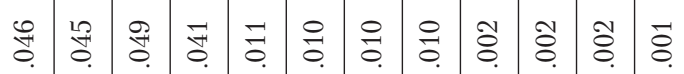

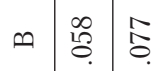

\&

Q

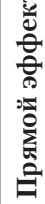

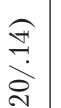

离

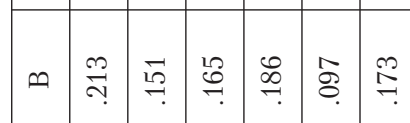

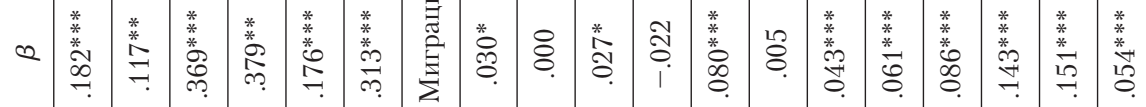

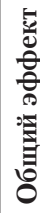

กิ $\stackrel{2}{2}$ 兽

幽 总

苍

๑ ㄱำ

\&

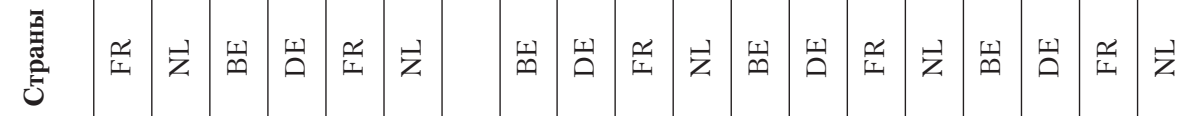

宣

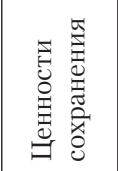

咅高

0
0
0
0
0
0
0
0
0
0

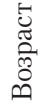




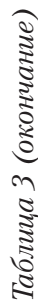

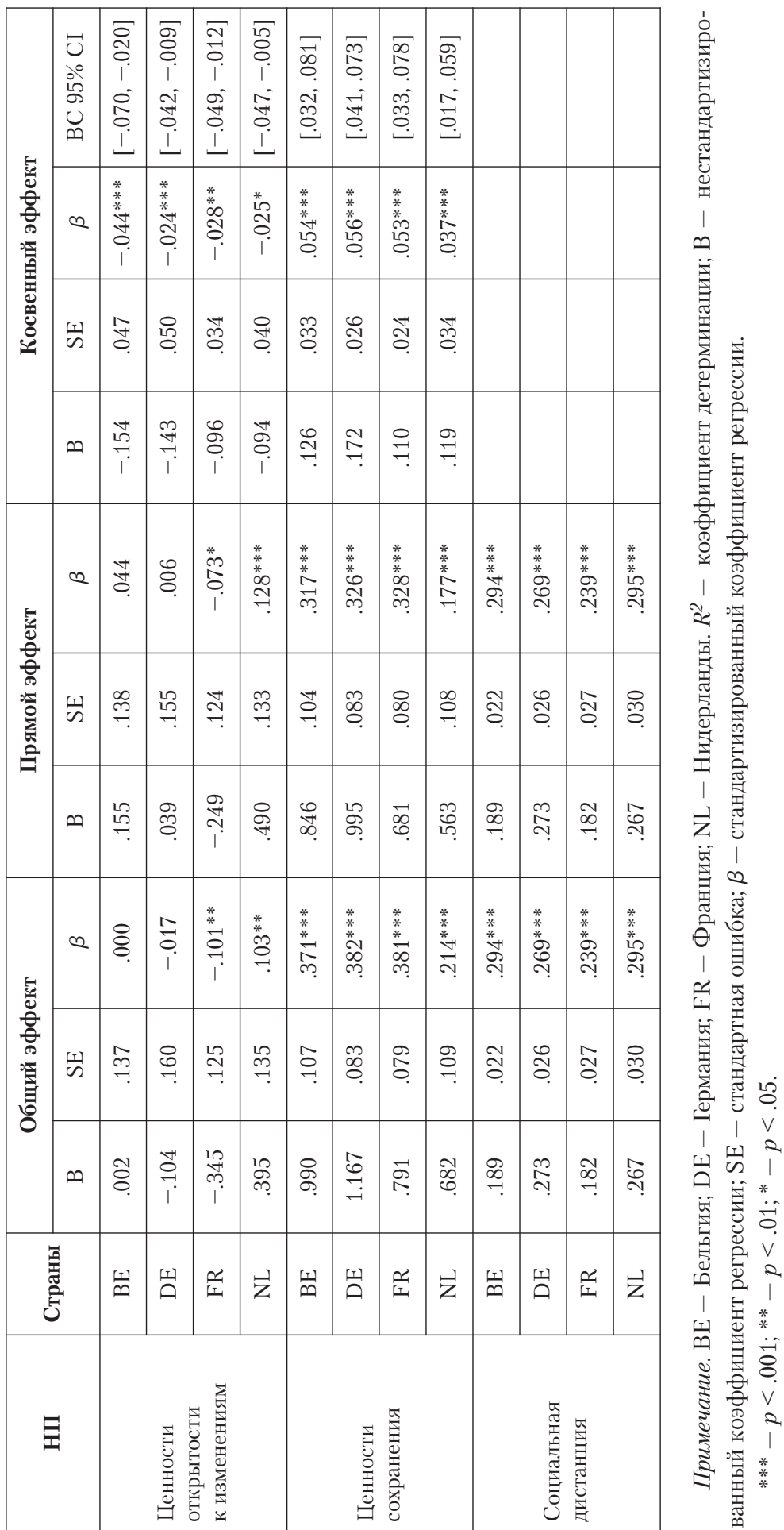


Рисунок 3

Проверяемые в анализе связи на путевой диаграмме

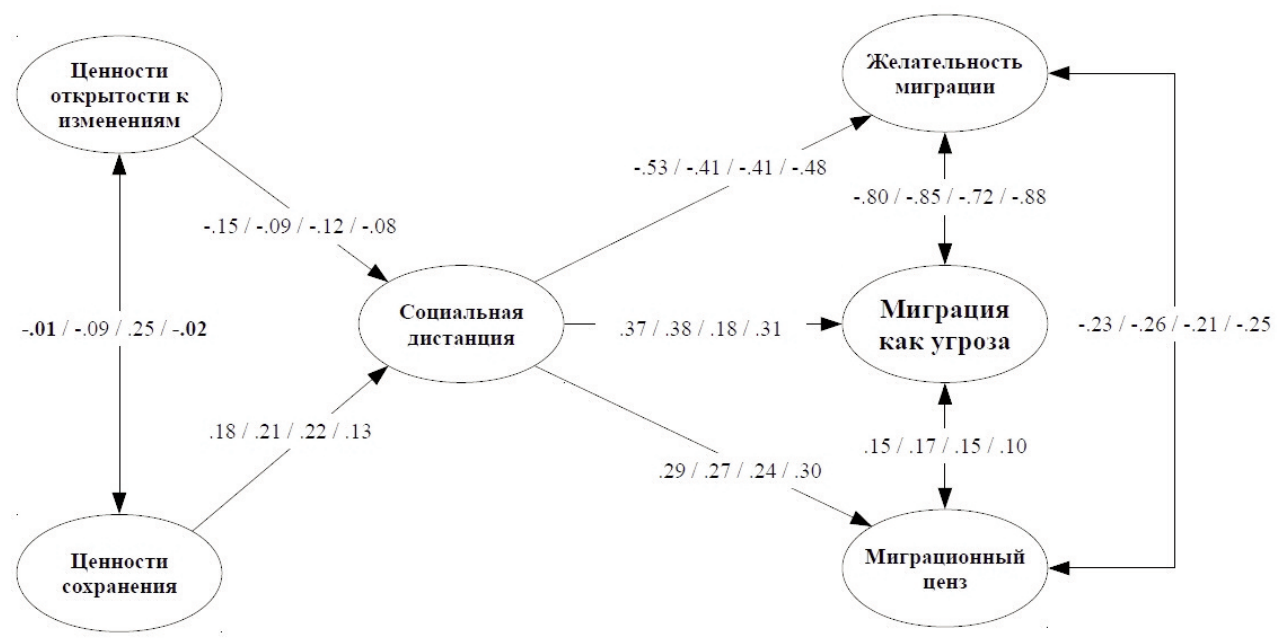

Примечание. Стандартизированные регрессионные коэффициенты, по порядку: Бельгия, Германия, Франция, Нидерланды. Все регрессионные коэффициенты значимы на уровне значимости $p<.05$, кроме выделенных полужирным шрифтом.

Размеры эффекта $f^{2}$ Коэна для зависимых переменных

Таблица 4

\begin{tabular}{|l|c|c|c|c|c|}
\hline \multirow{2}{*}{} & \multirow{2}{*}{$\mathbf{n}$} & \multicolumn{5}{|c|}{ Страны } \\
\cline { 3 - 6 } & & Бельгия & Германия & Франция & Нидерланды \\
\hline $\begin{array}{l}\text { Ценности открытости к } \\
\text { изменениям }\end{array}$ & 3 & .25 & .22 &. $\mathbf{0 5}$ & .18 \\
\hline Ценности сохранения & 3 &. $\mathbf{1 1}$ & $\mathbf{. 0 9}$ & $\mathbf{. 0 9}$ & $\mathbf{. 0 9}$ \\
\hline Социальная дистанция & 5 &. $\mathbf{1 0}$ & $\mathbf{. 1 1}$ & .15 &. $\mathbf{. 1 1}$ \\
\hline Желательность миграции & 6 & .47 & .35 & .39 & .32 \\
\hline Миграция как угроза & 6 & .16 & .25 &. $\mathbf{0 8}$ &. $\mathbf{. 1 2}$ \\
\hline Миграционный ценз & 6 & .28 & .28 & .25 & .16 \\
\hline & $N$ & 1618 & 2703 & 1650 & 1687 \\
\hline
\end{tabular}

Примечание. Полужирным шрифтом выделены малые размеры эффекта $f^{2}<.15 ; \mathrm{n}-$ кол-во предикторов; $\mathrm{N}$ - размер выборки. 
является эффект полной медиации социальной дистанцией некоторых связей между отдельными ценностями и отдельными установками на разных выборках.

\section{Обсуждение результатов}

Итак, в данном исследовании было показано, что связи между ценностями, социальной дистанцией и установками по отношению к миграции носят универсальный характер, однако общая картина свидетельствует о том, что специфика и характер рассматриваемых связей в целом несколько варьируются в разных культурах, на что в дальнейшем следует обратить внимание исследователям, которые занимаются кросскультурной проблематикой.

Поддержка определенных индивидуальных ценностей является важным элементом проводимой политики в отношении мигрантов (Citrin et al., 2014), отчасти механизм действия ценностей был показан в данном исследовании, но, так как ценности на индивидуальном уровне связаны с ценностями на культурном уровне (Dobewall, Strack, 2014), полностью механизм влияния ценностей еще предстоит раскрыть, связав индивидуальный и культурный уровень между собой. Так, например, утверждается, что коллективистские ценности связаны с негативными установками по отношению к мигрантам из-за тенденции к более сильному различию между ин- и аутгруппой, а носители гуманистических ценностей более чувствительны к трудностям, с которыми сталкиваются неблагополучные группы (Leong, Ward, 2006; Kim et al., 2015), на инди- видуальном уровне имеется сходная ситуация (Davidov, Meuleman, 2012; Davidov et al., 2014). И, несмотря на то что в данном исследовании, в отличие от ранее проведенных (см.: Davidov et al., 2008; Davidov, Meuleman, 2012; Davidov et al., 2014), рассматриваются ценности открытости к изменениям, полностью все ценности, описанные в теории Ш. Шварца, рассмотрены также не были.

Кроме того, недостатком данного исследования является тот факт, что при рассмотрении положительного отношения к миграции модель не включает в себя факторы, которые ему способствуют, так как отсутствие социальной дистанции само по себе не предполагает положительного отношения к миграции. При этом имеет смысл учитывать специфику для каждой конкретной группы мигрантов и особенности их адаптации, а не рассматривать как аутгруппу каких-то «абстрактных» мигрантов, поскольку из-за различной культурной дистанции разнообразные группы могут восприниматься по-разному (Григорьев, 2015а, 2015б, 2015в).

Нерешенной также является проблема объяснения различного отношения к мигрантам и миграционной политике, поэтому в обществе существуют одновременно и негативные и позитивные установки по отношению к мигрантам, но на сегодня для этого даже современные многоуровневые теории не имеют удовлетворительной объяснительной способности (Berg, 2015). Тем не менее в целом полученные в данном исследовании результаты можно считать полезными для понимания некоторых аспектов межкультурных отношений. 


\section{Литература}

Григорьев, Д. С. (2015а). Взаимосвязь выраженности этнической идентичности и аккультурационных установок мигрантов с уровнем их социоэкономической адаптации. Культурно-историческая психология, 11(1), 71-85.

Григорьев, Д. С. (2015б). Влияние выраженности этнической идентичности на выбор мигрантами их аккультурационной установки. Известия Саратовского университета. Новая серия. Серия: Акмеология образования. Психология развития, 4(1), 63-66.

Григорьев, Д. С. (2015в). Аккультурационные профили мигрантов и уровень их социоэкономической адаптации. Мир образования - образование в мире, 59(3), 75-85.

Berg, J. A. (2015). Explaining attitudes toward immigrants and immigration policy: A review of the theoretical literature: Theories of immigration attitudes. Sociology Compass, 9(1), 23-34.

Bogardus, E. S. (1925). Social distance and its origins. Journal of Applied Sociology, 9, 216-226.

Chen, F. F. (2007). Sensitivity of goodness of fit indexes to lack of measurement invariance. Structural Equation Modeling, 14, 464-504.

Citrin, J., Levy, M., \& Wright, M. (2014). Multicultural policy and political support in European democracies. Comparative Political Studies, 47(11), 1531-1557.

Davidov, E., \& Meuleman, B. (2012). Explaining attitudes towards immigration policies in European countries: The role of human values. Journal of Ethnic and Migration Studies, 38(5), 757-775.

Davidov, E., Meuleman, B., Billiet, J., \& Schmidt, P. (2008). Values and support for immigration: A cross-country comparison. European Sociological Reviere, 24, 583-599.

Davidov, E., Meulemann, B., Schwartz, S. H., \& Schmidt, P. (2014). Individual values, cultural embeddedness, and anti-immigration sentiments: Explaining differences in the effect of values on attitudes toward immigration across Europe. KZfSS Kölner Zeitschrift für Soziologie und Sozialpsychologie, 66(S1), 263-285.

Dobewall, H., \& Strack, M. (2014). Relationship of Inglehart's and Schwartz's value dimensions revisited: Relationship of value dimensions. International Journal of Psychology, 49(4), 240-248.

Duckitt, J. (2003). Prejudice and intergroup hostility. In D. Sears, L. Huddy, \& R. Jervis (Eds.), Oxford handbook of political psychology (pp. 559-600). Oxford: Oxford University Press.

ESS Round 7: European Social Survey Round 7 Data. (2014). Data file edition 1.0. Norwegian Social Science Data Services, Norway - Data Archive and distributor of ESS data for ESS ERIC.

ESS Round 7: European Social Survey. (2015). ESS-7 2014 Documentation Report. Edition 1.0. Bergen, European Social Survey Data Archive, Norwegian Social Science Data Services for ESS ERIC.

Fitzgerald, J., Curtis, K. A., \& Corliss, C. L. (2012). Anxious publics worries about crime and immigration. Comparative Political Studies, 45, 477-506.

Fornell, C., \& Lacker, D. (1981). Evaluating structural equation models with unobservable variables and measurement error. Journal of Marketing Research, 18(1), 39-50.

Hair, J., Black, W., Babin, B., \& Anderson, R. (2010). Multivariate Data Analysis (7th ed.). Upper Saddle River, NJ: Prentice-Hall, Inc.

Halperin, E., Canetti-Nisim, D., \& Pedahzur, A. (2007). Threatened by the uncontrollable: Psychological and socio-economic antecedents of social distance towards labor migrants in Israel. International Journal of Intercultural Relations, 31(4), 459-478.

Higgins, G. E., Gabbidon, S. L., \& Martin, F. (2010). The role of race/ethnicity and race relations on public opinion related to the immigration and crime link. Journal of Criminal Justice, 38(1), 51-56. 
Hu, L. T., \& Bentler, P. M. (1999). Cutoff criteria for fit indexes in covariance structure analysis: Conventional criteria versus new alternatives. Structural Equation Modeling, 6(1), 1-55.

Kadushin, C. (1962). Social distance between client and professional. American Journal of Sociology, 67(5), 517-531.

Kim, H. J., Yoo, H. Y., \& Chung, Y. K. (2015). Social distance towards the North Korean refugees in South Korean society. Korea Observer, 46(2), 295-320.

Kleinpenning, G., \& Hagendoorn, L. (1993). Forms of racism and the cumulative dimension of ethnic attitudes. Social Psychology Quarterly, 56(1), 21-36.

Laumann, E. O. (1965). Subjective social distance and urban occupational stratification. American Journal of Sociology, 71(1), 26-36.

Lee, T. L., \& Fiske, S. T. (2006). Not an outgroup, not yet an ingroup: Immigrants in the stereotype content model. International Journal of Intercultural Relations, 30(6), 751-768.

Leong, C.-H. (2008). A multilevel research framework for the analyses of attitudes toward immigrants. International Journal of Intercultural Relations, 32(2), 115-129.

Leong, C.-H., \& Ward, C. (2006). Cultural values and attitudes toward immigrants and multiculturalism: The case of the Eurobarometer survey on racism and xenophobia. International Journal of Intercultural Relations, 30(6), 799-810.

Malhotra, N. K., \& Dash, S. (2010). Marketing research: An applied orientation (6th ed.). Upper Saddle River, NJ [etc.]: Pearson Education.

Marger, M. N. (1994). Race and ethnic relations: American and global perspectives (3rd ed.). Belmont, CA: Wadsworth.

Migration and Migrant Population Statistics. (2015, May). Retrieved from http://ec.europa.eu/eurostat/statistics-explained/index.php/Migration_and_migrant_population_statistics

Milfont, R., \& Fischer, R. (2010). Testing measurement invariance across groups: Applications in cross-cultural research. International Journal of Psychological Research, 3, 112-131.

Park, R. E. (1924). The concept of social distance as applied to the study of racial attitudes and racial relations. Journal of Applied Sociology, 8, 339-344.

Pettigrew, T. (2003). People under threat: Americans, Arabs and Israelis. Peace and Conflict:Journal of Peace Psychology, 9(1), 69-90.

Sagiv, L., \& Schwartz, S. H. (1995). Value priorities and readiness for out-group social contact.Journal of Personality and Social Psychology, 69, 437-448.

Schwartz, S. H., \& Butenko, T. P. (2014). Values and behavior: Validating of refined values theory in Russia. European Journal of Social Psychology, 44(7), 799-813.

Schwartz, S. H., Cieciuch, J., Vecchione, M., Davidov, E., Fischer, R., Beierlein, C., ... Konty, M. (2012). Refining the theory of basic individual values. Journal of Personality and Social Psychology, 103, 663-688.

Simmel, G. (1950). The sociology of Georg Simmel. New York: Free Press.

Simon, R. J., \& Sikich, K. W. (2007). Public attitudes toward immigrants and immigration policies across seven nations. International Migration Reviere, 41, 956-962.

Sniderman, P. M., Hagendoorn, L., \& Prior, M. (2004). Predisposing factors and situational triggers: Exclusionary reactions to immigrant minorities. American Political Science Reviere, 98, 35-49.

Stephan, W. G., \& Stephan, C. W. (2001). Improving intergroup relations. Dubuque, IA: Brown \& Benchmark. 
Tartakovsky, E., \& Walsh, S. D. (2016). Testing a new theoretical model for attitudes toward immigrants: The case of social workers attitudes toward asylum seekers in Israel. Journal of CrossCultural Psychology, 47(1), 72-96.

Vandenberg, R. J., \& Lance, C. E. (2000). A review and synthesis of the measurement invariance literature: Suggestions, practices, and recommendations for organizational research. Organizational Research Methods, 3(1), 4-70.

Weaver, C. N. (2008). Social distance as a measure of prejudice among ethnic groups in the United States. Journal of Applied Social Psychology, 38(3), 779-795.

Williams, R. M., Jr. (1964). Strangers next door: Ethnic relations in American communities. Englewood Cliffs, NJ: Prentice Hall.

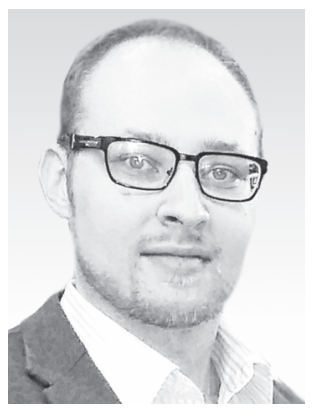

Григорьев Дмитрий Сергеевич - аспирант и стажер-исследователь, Международная научно-учебная лаборатория социокультурных исследований, Экспертный институт, Национальный исследовательский университет «Высшая школа экономики».

Сфера научных интересов: межэтнические отношения, социальная идентичность, социальные представления, психология аккультурации, кросс-культурная психология.

Контакты: dgrigoryev@hse.ru

\title{
Values, Social Distance and Attitudes toward Immigration: A Cross- Cultural Study of Belgium, Germany, France and the Netherlands
}

\author{
Dmitry S. Grigoryev
}

${ }^{a}$ National Research University Higher School of Economics, 20 Myasnitskaya str., Moscow, 101000, Russian Federation

\begin{abstract}
The article addresses the relationship between individual values, social distance and positive and negative attitudes toward immigration. The objective of the present study is the verification of the universal character of these relationship. Previously the researchers very rarely addressed both aspects of attitudes toward immigrants: appraisal of immigrants by the host society and attitudes of the host society toward the migration policy. In the present study both these aspects were considered in the form of social distance and three attitudes toward immigration. With the data analysis of the 7th round from the European Social Survey (ESS), 2014 for four European countries (Belgium, Germany, France and the Netherlands), after the test of metric (strong factorial) invariance of the scales and its reliability, with the use of structural equation modeling it was found that, as it was supposed in the hypotheses of the study, social distance is positively
\end{abstract}


associated to the conservation values and negatively associated with the openness to change, and positively associated to negative attitudes toward immigration (immigration as a threat and necessity of some immigration qualification) and negatively associated with positive attitudes (desirability of immigration). These relationship was universal, however many relationship that were cultural-specific. Especially notable is the effect of full mediation in different samples of some relationship between some values and attitudes by social distance. The obtained results are valuable for understanding of some aspects of intercultural relations.

Keywords: social distance, values, interethnic relationships, immigration, cross-cultural psychology.

\section{References}

Berg, J. A. (2015). Explaining attitudes toward immigrants and immigration policy: A review of the theoretical literature: Theories of immigration attitudes. Sociology Compass, 9(1), 23-34.

Bogardus, E. S. (1925). Social distance and its origins. Journal of Applied Sociology, 9, 216-226.

Chen, F. F. (2007). Sensitivity of goodness of fit indexes to lack of measurement invariance. Structural Equation Modeling, 14, 464-504.

Citrin, J., Levy, M., \& Wright, M. (2014). Multicultural policy and political support in European democracies. Comparative Political Studies, 47(11), 1531-1557.

Davidov, E., \& Meuleman, B. (2012). Explaining attitudes towards immigration policies in European countries: The role of human values. Journal of Ethnic and Migration Studies, 38(5), 757-775.

Davidov, E., Meuleman, B., Billiet, J., \& Schmidt, P. (2008). Values and support for immigration: A cross-country comparison. European Sociological Review, 24, 583-599.

Davidov, E., Meulemann, B., Schwartz, S. H., \& Schmidt, P. (2014). Individual values, cultural embeddedness, and anti-immigration sentiments: Explaining differences in the effect of values on attitudes toward immigration across Europe. KZfSS Kölner Zeitschrift für Soziologie und Sozialpsychologie, 66(S1), 263-285.

Dobewall, H., \& Strack, M. (2014). Relationship of Inglehart's and Schwartz's value dimensions revisited: Relationship of value dimensions. International Journal of Psychology, 49(4), 240-248.

Duckitt, J. (2003). Prejudice and intergroup hostility. In D. Sears, L. Huddy, \& R. Jervis (Eds.), Oxford handbook of political psychology (pp. 559-600). Oxford: Oxford University Press.

ESS Round 7: European Social Survey Round 7 Data. (2014). Data file edition 1.0. Norwegian Social Science Data Services, Norway - Data Archive and distributor of ESS data for ESS ERIC.

ESS Round 7: European Social Survey. (2015). ESS-7 2014 Documentation Report. Edition 1.0. Bergen, European Social Survey Data Archive, Norwegian Social Science Data Services for ESS ERIC.

Fitzgerald, J., Curtis, K. A., \& Corliss, C. L. (2012). Anxious publics worries about crime and immigration. Comparative Political Studies, 45, 477-506.

Fornell, C., \& Lacker, D. (1981). Evaluating structural equation models with unobservable variables and measurement error. Journal of Marketing Research, 18(1), 39-50.

Grigoryev, D. S. (2015a). Relationship between Ethnic identification and acculturation attitudes of immigrants with their level of socio-economic adaptation. Cultural-Historical Psychology, 11(1), $71-85$. 
Grigoryev, D. S. (2015b). Effect of ethnic identification on the choice of acculturation attitudes of immigrants. Izvestiya Saratovskogo Universiteta. Novaya Seriya. Seriya: Akmeologiya Obrazovaniya. Psikhologiya Razvitiya, 4(1), 63-66.

Grigoryev, D. S. (2015c). Acculturation profiles of immigrants and their level of socio-economic adaptation. Mir Obrazovaniya - Obrazovanie v Mire, 59(3), 75-85.

Hair, J., Black, W., Babin, B., \& Anderson, R. (2010). Multivariate Data Analysis (7th ed.). Upper Saddle River, NJ: Prentice-Hall, Inc.

Halperin, E., Canetti-Nisim, D., \& Pedahzur, A. (2007). Threatened by the uncontrollable: Psychological and socio-economic antecedents of social distance towards labor migrants in Israel. International Journal of Intercultural Relations, 31(4), 459-478.

Higgins, G. E., Gabbidon, S. L., \& Martin, F. (2010). The role of race/ethnicity and race relations on public opinion related to the immigration and crime link. Journal of Criminal Justice, 38(1), 51-56.

Hu, L. T., \& Bentler, P. M. (1999). Cutoff criteria for fit indexes in covariance structure analysis: Conventional criteria versus new alternatives. Structural Equation Modeling, 6(1), 1-55.

Kadushin, C. (1962). Social distance between client and professional. American Journal of Sociology, 67(5), 517-531.

Kim, H. J., Yoo, H. Y., \& Chung, Y. K. (2015). Social distance towards the North Korean refugees in South Korean society. Korea Observer, 46(2), 295-320.

Kleinpenning, G., \& Hagendoorn, L. (1993). Forms of racism and the cumulative dimension of ethnic attitudes. Social Psychology Quarterly, 56(1), 21-36.

Laumann, E. O. (1965). Subjective social distance and urban occupational stratification. American Journal of Sociology, 71(1), 26-36.

Lee, T. L., \& Fiske, S. T. (2006). Not an outgroup, not yet an ingroup: Immigrants in the stereotype content model. International Journal of Intercultural Relations, 30(6), 751-768.

Leong, C.-H. (2008). A multilevel research framework for the analyses of attitudes toward immigrants. International Journal of Intercultural Relations, 32(2), 115-129.

Leong, C.-H., \& Ward, C. (2006). Cultural values and attitudes toward immigrants and multiculturalism: The case of the Eurobarometer survey on racism and xenophobia. International Journal of Intercultural Relations, 30(6), 799-810.

Malhotra, N. K., \& Dash, S. (2010). Marketing research: An applied orientation (6th ed.). Upper Saddle River, NJ [etc.]: Pearson Education.

Marger, M. N. (1994). Race and ethnic relations: American and global perspectives (3rd ed.). Belmont, CA: Wadsworth.

Migration and Migrant Population Statistics. (2015, May). Retrieved from http://ec.europa.eu/eurostat/statistics-explained/index.php/Migration_and_migrant_population_statistics

Milfont, R., \& Fischer, R. (2010). Testing measurement invariance across groups: Applications in cross-cultural research. International Journal of Psychological Research, 3, 112-131.

Park, R. E. (1924). The concept of social distance as applied to the study of racial attitudes and racial relations. Journal of Applied Sociology, 8, 339-344.

Pettigrew, T. (2003). People under threat: Americans, Arabs and Israelis. Peace and Conflict:Journal of Peace Psychology, 9(1), 69-90.

Sagiv, L., \& Schwartz, S. H. (1995). Value priorities and readiness for out-group social contact.Journal of Personality and Social Psychology, 69, 437-448.

Schwartz, S. H., \& Butenko, T. P. (2014). Values and behavior: Validating of refined values theory in Russia. European Journal of Social Psychology, 44(7), 799-813. 
Schwartz, S. H., Cieciuch, J., Vecchione, M., Davidov, E., Fischer, R., Beierlein, C., ... Konty, M. (2012). Refining the theory of basic individual values. Journal of Personality and Social Psychology, 103, 663-688.

Simmel, G. (1950). The sociology of Georg Simmel. New York: Free Press.

Simon, R. J., \& Sikich, K. W. (2007). Public attitudes toward immigrants and immigration policies across seven nations. International Migration Review, 41, 956-962.

Sniderman, P. M., Hagendoorn, L., \& Prior, M. (2004). Predisposing factors and situational triggers: Exclusionary reactions to immigrant minorities. American Political Science Reviere, 98, 35-49.

Stephan, W. G., \& Stephan, C. W. (2001). Improving intergroup relations. Dubuque, IA: Brown \& Benchmark.

Tartakovsky, E., \& Walsh, S. D. (2016). Testing a new theoretical model for attitudes toward immigrants: The case of social workers attitudes toward asylum seekers in Israel. Journal of CrossCultural Psychology, 47(1), 72-96.

Vandenberg, R. J., \& Lance, C. E. (2000). A review and synthesis of the measurement invariance literature: Suggestions, practices, and recommendations for organizational research. Organizational Research Methods, 3(1), 4-70.

Weaver, C. N. (2008). Social distance as a measure of prejudice among ethnic groups in the United States. Journal of Applied Social Psychology, 38(3), 779-795.

Williams, R. M., Jr. (1964). Strangers next door: Ethnic relations in American communities. Englewood Cliffs, NJ: Prentice Hall.

Dmitry S. Grigoryev - Ph.D. student, researcher, International scientific-educational laboratory for socio-cultural research of the Expert Institute, National Research University Higher School of Economics.

Research areas: interethnic relations, social identity, social representations, psychology of acculturation, cross-cultural psychology.

E-mail: dgrigoryev@hse.ru 